\title{
FTOR-ModPSO: A Fault Tolerance and an Optimal Relay Node Selection Algorithm for Wireless Sensor Networks using Modified PSO
}

POGULA SREEDEVI ( $\sim$ sreedevipogula37@gmail.com )

Koneru Lakshmaiah Education Foundation

\section{S. Venkateswarlu}

Koneru Lakshmaiah Education Foundation

\section{Research Article}

Keywords: Fault tolerance, Backup Cluster heads, Inter-cluster data aggregation, Aggregator nodes, Energy Consumption.

Posted Date: March 7th, 2022

DOI: https://doi.org/10.21203/rs.3.rs-207928/v1

License: (c) (1) This work is licensed under a Creative Commons Attribution 4.0 International License. Read Full License 


\section{FTOR-ModPSO: A Fault Tolerance and an Optimal Relay Node Selection Algorithm for Wireless Sensor Networks using Modified PSO}

\author{
Pogula Sreedevi ${ }^{1}$ \\ Research Scholar, Department of \\ Computer Science Engineering, Koneru \\ Lakshmaiah Education Foundation, \\ Vaddeswaram, Andhra Pradesh, India \\ and Working as an Assistant professor \\ in "G Pulla Reddy Engineering College", \\ Kurnool, AP, India. \\ Email: sreedevipogula37@gmail.com
}

\author{
Dr. S. Venkateswarlu² \\ Research Supervisor, Department of \\ Computer Science Engineering, Koneru \\ Lakshmaiah Education Foundation \\ Vaddeswaram, Andhra Pradesh, India. \\ Email: somu23@kluniversity.in
}

\section{$\underline{\text { Abstract: }}$}

Most of the wireless sensor networks are energy limited. To improve the lifetime of a network, the energy consumption should be reduced. Clustering is a proven technique that reduces energy consumption in WSNs. The energy can be saved by sending the collected information to the sink using the Cluster Head $(\mathrm{CH})$ nodes. Fault tolerance is a crucial issue in WSNs, and one cluster head failure collapses the entire data communication. This paper presents a fault tolerance cluster-based routing technique. This paper aims to provide improved fault tolerance and data aggregation technique for clustered WSN using FTOR-ModPSO (Fault tolerance and optimal relay node with modified Particle Swarm Optimization). Here, the fault tolerance is achieved by selecting the backup cluster head $(\mathrm{BKCH})$ for every cluster. The method consists of two phases. The network is categorized into clusters in the first phase whereas in the second phase, $\mathrm{CHs}$ and $\mathrm{BKCHs}$ are selected. Intracluster communication happens between member and $\mathrm{CH}$ nodes. Aggregator nodes (AG) are used for inter- cluster communication, and modified PSO identifies $\mathrm{CH}$ and AG's best relay. The fault tolerance and packet delivery rate are increased with less energy utilization compared to other existing energy-saving protocols by implementing this method.

Keywords: Fault tolerance, Backup Cluster heads, Inter-cluster data aggregation, Aggregator nodes, Energy Consumption.

\section{Introduction}

A significant role is played by WSNs in the $21^{\text {st }}$ century [1]. A huge amount of increasingly significant applications is run by WSN. The deployment of lower cost, batterypowered sensor nodes is done physically in a region for collecting beneficial data as well as transmitting it via wireless links towards the sink nodes. Communication is performed within the sensors in a direct or an indirect way with the sink. Controlled energy 
resources are allotted to the nodes where the major issues of WSNs are represented. The energy is conserved, and the lifespan of the network is prolonged by considering the significant factors such as energy effectiveness, robust self-association, clustering, as well as routing protocols which ensure appropriate network operations. Also, a little imperfection is presented by WSNs due to the respective constrained buffering characteristics in addition to the computing sources. Fig. 1 represents the architecture of problem formulation in WSN.

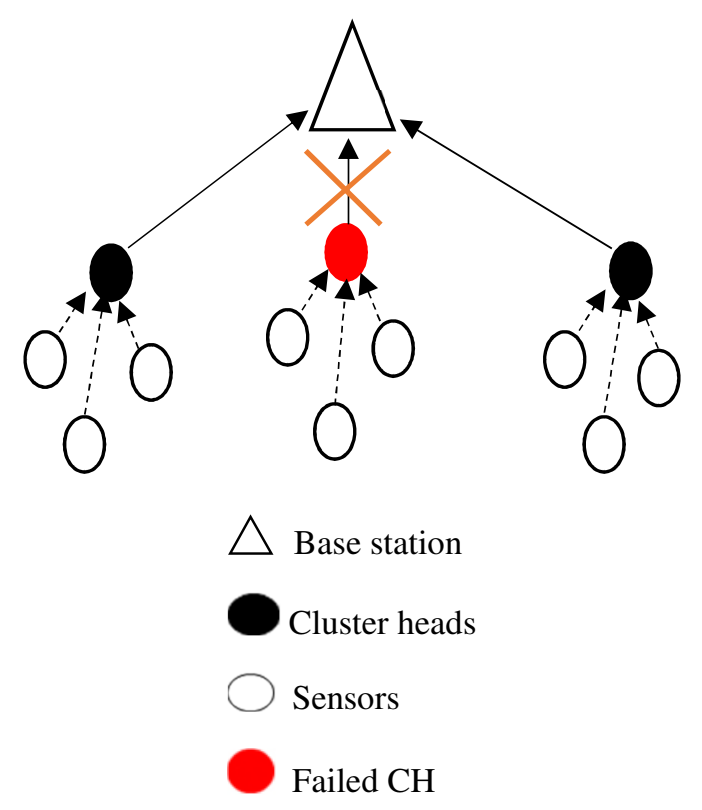

Fig. 1. CH failure and interruption in data communication.

Direct communication by the sink node for longer distances [2], [3], [4], [5], [6] is inhibited by the constrained energy as well as the communicating abilities of a sensor node. Thus, a multihop communication model is followed by the transmission of information from the source sensor node towards the sink node. Establishing a new energy-balancing routing path where network lifespan is increased is the major issue in this situation. A quality of service (QoS) guarantee [7-11], [12], [13] is required by several missioncritical and real-time implementations besides the increment in the lifetime of the network. Moreover, safety is a major challenge in WSNs since the neglected activities as well as the undependable render sensor nodes are exposed to attacks [3], [1213], [14-15].

The presentation of several power-saving approaches was made during the last decades. The author has begun the investigation of the physical layer towards the link layer as well as later towards the network routing layer on the basis of the data-accessing schemes [16]. Several authors have proposed various clustering protocols [17]. Setup phase as well as steady state phase are the two techniques obtained by separating data sensing, aggregation, and transmitting phases. The data is systematically perceived as well as relayed by the cluster member nodes to a $\mathrm{CH}$. The distribution of wireless media is conducted among several nodes within the 
cluster. The scheduling of the data aggregation is established by the $\mathrm{CH}$ and is conveyed to the overall participant nodes. A TDMA access method is used to implement the aggregation procedure. The data reduction is realized by the $\mathrm{CH}$ and the compression procedures for decreasing the size of the data as well as progressing to the BS is applied.

The disadvantages of reliable and energyefficient multi-hop LEACH-based clustering protocol [26] for WSNs is the increase in the routing overhead because of the multi-hop routing method, in addition to increased reclustering with the increase in communication. The drawbacks in ant colony optimization (ACO)-based QoS aware energy balancing secure routing procedure [27] for WSNs are that it doesn't require the repairing technique when a path fails, and routing overhead is increased in this approach because of the presence of several metrics in selecting a route. The recovery technique during a $\mathrm{CH}$ failure is not required and the overhead is increased due to the presence of several factors in selecting a $\mathrm{CH}$ within the energy-efficient QoS-aware intelligent hybrid clustered routing protocol [28] which are the drawbacks to this approach. The drawbacks in probabilitybased cluster head selection and fuzzy multipath-routing for extending the lifespan of WSNs [29] are the occurrence of path failure by ignoring the construction of the routing path and the absence of a recovery technique when a path failure occurs.

To address the aforementioned issues, here we introduce a new fault tolerance technique with improved data aggregation and relay selection technique. Two different phases involve in the proposed technique such as fault tolerance routing and fault tolerance clustering. By using criteria including delay between the number of neighbors and nodes, probability value, distance to $\mathrm{BS}$, and residual energy, the selection of $\mathrm{CHs}$ is made in the first phase. In the setup phase, BKCHs are chosen in addition to the CHs. Two levels are included in the fault tolerant routing such as inter-cluster routing and intra-cluster routing. The data from the member nodes is aggregated by $\mathrm{CH}$ in intra-cluster routing. By using PSO functions, the efficient path among $\mathrm{CHs}$ towards the aggregator node is estimated in inter-cluster routing.

The proposed mechanism provides fault tolerance in the following two conditions. 1. Fault tolerance when $\mathrm{CH}$ fails. 2. Fault tolerance when the route fails. When the $\mathrm{CH}$ fails, the data transmission occurring in the network is disturbed and there are huge 
chances of the data being dropped without reaching their estimated destination. This will lead to serious problems where the data are sensitive and need to be processed on time, so there must be a solution to address this issue. Also, data transmission can be disturbed by transmitting the data packets through inappropriate relay nodes. Our proposed fault tolerance mechanism addresses these issues using backup CHs and efficient relay node selection. The backup $\mathrm{CHs}$ are selected using multiple metrics like residual energy, distance to $\mathrm{BS}$, probability value, delay between the nodes and number of neighbors. To reduce the overhead caused by data aggregation in every $\mathrm{CHs}$, our method introduces a special node called Aggregator for data aggregation. Also, to manage the route failures during data transmission, the FTOR-ModPSO ensures the fair selection of relay nodes using fitness evaluation of the nodes.

The contribution and advantages of the proposed protocol:

- The backup CHs are selected using multi-level parameters such as distance, energy and delay between the nodes.

- Data aggregation performs by the Aggregator nodes for every $\mathrm{CH}$, so the overhead incurred to $\mathrm{CHs}$ due to aggregation is drastically reduced.

- The minimized overhead optimized the energy utilization rate in both sensor nodes and their respective CHs.

- The appropriate relay node selection mechanism ensures a reliable path between the communicating nodes; therefore, the possibility of data drops due to route failure is avoided.

- This improves the overall data delivery rate and energy utilization in the sensor nodes.

- The proposed method provides fault tolerance in case of $\mathrm{CH}$ failure and ensures an uninterrupted data transmission using backup CHs.

- Inter-cluster data aggregation is improved using Aggregator nodes with reduced energy consumption.

- A modified PSO algorithm selects the suitable relay nodes and reduces the communication overhead.

- Using the fault tolerance mechanism, the data delivery rate and energy utilization is improved.

In sum, the scope of this paper is providing a light-weight and effective fault tolerance to 
WSNs by introducing and backing up $\mathrm{CHs}$ as fault tolerance for clustering where the data are aggregated by special Aggregator nodes which reduce the overhead at the SINK level. Data communication is managed by PSO and TDMA is used for further data scheduling between the nodes during data aggregation.

The remaining part of the paper is organized as follows: The relevant research for the proposed work is explored in Section 2. The proposed protocol architecture, the TDMA schedule, modified PSO relay selection, routing phase (intra-cluster routing and intercluster routing), redesigned $\mathrm{CH}$ selection criteria, and clustering phase have been described in Section 3. The proposed approach with experimental results discusses in Section 4 and finally, the proposed work concludes in Section 5.

\section{Literature survey}

The advantages of node energy heterogeneousness within WSN by the EEHC (energy-efficient heterogeneous clustered) protocol design is considered by Kumar et al., [18] on behalf of a tri-level network. A $\mathrm{CH}$ is selected by it depending upon the residual energy of sensor node via a probability threshold operation. EEHC is outperformed by means of a heterogeneous approach compared to $\mathrm{LEACH}$ with respect to the enhancement of the lifetime of the network. Moreover, an energy model is proposed by Sharma et al., [19] and a traffic \& energy-aware routing (TEAR) is presented to refine the stability interval if assumes that the arbitrary initial energy includes in the sensor nodes. The limitations of the complication in the systems is prevailed by the variations in the origination rate of traffic.

Dutt et al., [20], Tanwar et al., [21, and Hong et al., [22] have been proposed Efficient and Dynamic Clustering Scheme (EDCS), Automata-based Multilevel Heterogeneous Routing (LA-MHR), and Cluster-head Restricted Energy-Efficient Protocol (CREEP) methods correspondingly as a part of the heterogeneous clustering networks. The reduction of several $\mathrm{CHs}$ for one round is reduced by CREEP for improving the lifetime of the network using the 2-stage heterogeneity. The lifespan of the network is upgraded by the outcome of the comparison between the mobile and the stationary HWSN situations. A multilevel heterogeneous node model depending on automatic learning is introduced by LAMHR. The employment of an S-model dependent learning procedure is done by selecting a $\mathrm{CH}$ while operating LA-MHR. Moreover, the $\mathrm{CHs}$ are elected by assigning the cognitive radio spectrum by the BS. The 
lifespan of the network is evaluated at the end by considering the whole problem.

A cluster-tree topology control based on the energy forecast (CTEF) was developed by Hong et al., [23] on behalf of network load balancing in addition to the savage of the energy when the various features such as PLR and link reliability are considered. The application of a central theorem as well as log normal distribution techniques is made by an accurate prediction of the mean energy of the network along with a traditional $\mathrm{CH}$ selection technique. The cluster formation in regard to the variation between the ideal and actual average residual energy is determined.

An energy-aware QoS routing protocol (EQRP) clustering method is proposed by Faheem and Gungor [24]. The effective bird mating optimization (BMO) impacted this approach on the basis of the reliable framework. The reliability of the network and the throughput is enhanced, the excess packet retransmission is reduced, the packet delivery ratio is improved, and the end-to-end delay is reduced by this routing protocol.

An intelligent modified chain model is presented by Mishra et al. [25]. Increasing the lifespan of the network is the main objective of this method which is obtained using PEGASIS with the selection of a $\mathrm{CH}$ nearer to a BS. Moreover, the transmission of data is done for BS via the participants of the overlapping chain technique.

AI-Sodairi et al., analyzed the efficiency of low-energy adaptive clustering hierarchy (LEACH) as well as LEACH-based protocols within the extension of the energyconstrained lifespan of WSNs. Energy consumption is reduced and balanced by proposing an increased LEACH clustering protocol named enhanced multi-hop LEACH which allows the enhanced packet delivery in addition to the lifespan of the network within WSNs. Moreover, the shortcoming of LEACH protocol is presented in this approach. Initially, the novel rules to select the $\mathrm{CH}$ in addition to the round time calculating depending upon residual energy is introduced in this approach. Later, the integration of multi-hop communication is done within WSN with the help of two operating techniques such as leveling and generic multi-hop routing [26].

Rathee et al., [27] proposed a QoS-aware and energy-balancing secure routing (QEBSR) technique with the help of ACO for providing an additional integrated overview of WSNs which was the development of the enhanced heuristics to calculate the end-to-end delay of 
transmission in addition to the trust factor of nodes upon a routing path.

Singh et al., proposed a multi-objective clustering scheme [28] to achieve the optimized consumption of energy, lifespan of a network, network throughput in addition to optimizing the delay of the network. The formulation of a fitness function is made for the heterogeneous and homogenous WSNs. An optimal $\mathrm{CH}$ is selected by using the fitness function for energy reduction in addition to the $\mathrm{CH}$ balancing. Depending upon the fitness function, the introduction of a novel hybrid clustered routing protocol.

Robinson et al., proposed a novel poweraware routing technique [29] on behalf of WSNs dependent on the threshold rate and fuzzy logic with a view to enhance the effectiveness of energy. The selection of $\mathrm{CHs}$ is made on the basis of the probability values of each node within WSN, and these are computed out of a single node's residual energy. The mean energy of the overall network at the present stage is calculated by using the cumulative residual node energy. Using multi-hop communication, fuzzy control is used to send the collected data out of the $\mathrm{CH}$ towards sink. The three factors considered by fuzzy control are the node's queue length, the distance between a node and BS, and the residual energy of the node.

From the above literature surveys, we observe that there is lack of a reliable fault tolerance technique which can balance the trade-off between fault tolerance and efficient routing. Some of the previously used fault tolerance protocols performed well in providing efficient fault tolerance in faulty environments but failed to address the issue of efficient data communication by selecting the optimal relay nodes, resulting in excess and unwanted consumption of resources. Here, in this paper, we provided an efficient solution that takes care of this trade-off. In this work, the fault tolerance in case of $\mathrm{CH}$ failure is handled by the selected BKCHs and a modified particle swarm optimizer is used for efficient relay selection that chooses the relay nodes based on node fitness evaluation parameters.

\section{System model}

Problems emerge while providing fault tolerance-based routing because of the limitations that exist in WSNs. Constrained battery capability, undefined locations, abundance, and a random scattering of nodes are considered specific issues in designing the routing protocol. Here we introduce the FTOR-ModPSO as proposed model. Fault 
tolerance clustering and fault tolerance routing are the two stages in this approach. The selection of $\mathrm{CHs}$ is made in this initial stage based on certain factors such as residual energy, distance to BS, probability value, the delay between the nodes, and the number of neighbors. The selection of $\mathrm{BKCHs}$ is done along with $\mathrm{CHs}$ within the setup phase. Intracluster routing and inter-cluster routing are included in the fault-tolerant routing within the 2nd stage. $\mathrm{CH}$ aggregates the data out of the participant nodes within the intra-cluster routing. Moreover, the PSO functions are used to determine the effective path amongst $\mathrm{CHs}$ within the inter-cluster routing. Figure 2 represent the block diagram of proposed system.

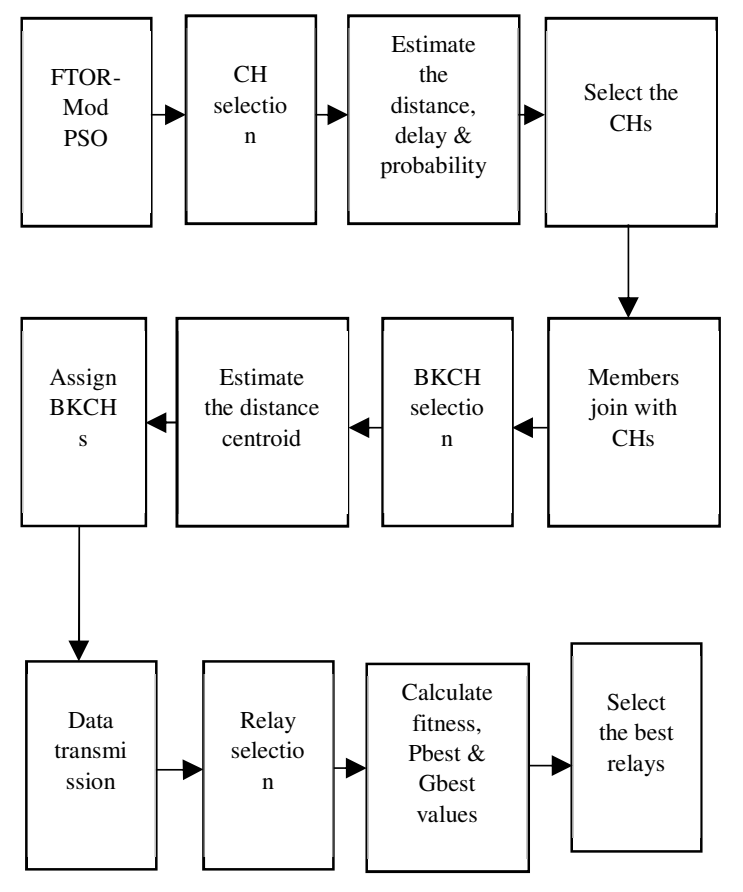

Fig. 2. Block diagram of proposed system

\subsection{Clustering phase:}

Dividing the network nodes within the clusters and assigning a $\mathrm{CH}$ on behalf of every $\mathrm{CH}$ receiver is the main objective of this phase. The data is later combined by the nodes and is transmitted towards the destination. Distance to BS, residual energy, probability value, the delay between the nodes, and neighbors' number are the parameters considered in selecting a $\mathrm{CH}$. Therefore, the selection of $\mathrm{CH}$ is made when a single node fulfills the selection parameters. The following equation (1) is used to calculate the distance among the nodes as well as BS:

$$
\begin{array}{r}
\text { Dist }_{i, \text { sink }}= \\
\sqrt{\left(X_{\operatorname{sink}}-X_{i}\right)^{2}+\left(Y_{\operatorname{sink}}-Y_{i}\right)^{2}}
\end{array}
$$

Where $X_{\text {sink }}, Y_{\text {sink }}$ and $X_{i}, Y_{i}$ indicate the locations of BS and node respectively.

In the network area, the energy consumption pattern of common sensor node and cluster head are different. The common nodes are transmitting the sensed data to their own cluster heads through their own slots. The constant data length $L$ is used for transmission. Therefore, the energy consumption of common node on transmitting the data is given by equation (2),

$$
\operatorname{Energy}_{(n)}=L * E_{\text {elec }}+L * \epsilon_{f s} * D_{c h}
$$


Here, L is data length, $E_{\text {elec }}$ is the expected energy dissipation by the radios in transmit or receive mode, $\epsilon_{f s}$ is the acceptable bit error rate based on data length $\mathrm{L}$ and $D_{c h}$ is the distance from the sensor node to its $\mathrm{CHs}$

The sensor nodes' average residual energy during the current round is calculated based on the following equation (3)

$$
E(r e s)=\frac{\sum \operatorname{Energy}(n)}{N N}
$$

Where, $\sum \operatorname{Energy}(n)=$ total energy of the sensor nodes and NN is the total number of alive nodes in the network.

The BKCH nodes are selected based on these conditions: Centroid of the nodes and the probability value between $0 \& 1$. The nodes (other than the previously selected $\mathrm{CH}$ nodes) which satisfy these conditions are selected as $\mathrm{BKCH}$.

The center relevant to each cluster is determined in this stage. On the basis of distance from the centroid, Backup Cluster Head is selected. WSN of nodes are assumed by $\mathrm{x}$ which is divided into $\mathrm{n}$ clusters $\left\{c_{1}, c_{2}, \ldots . c_{n}\right\}$ such that $c_{1} \cup c_{2} \cup \ldots . c_{k}=$ $\left\{x_{1}, x_{2}, \ldots x_{k}\right\}$ and $c_{i} \cap c_{j}=\Omega$. In equation (4), the center of cluster groups or mean is assumed by $c_{n}$.

$$
c_{n}=\frac{1}{N} \sum_{x_{i} \in c_{k}} x_{i}
$$

The minimization of total Euclidean distance between cluster members and backup cluster head is the objective function of determining the centroid. The minimum distance formulae algorithm is used in this process that helps to minimize the sum of distances from each sensor node to its cluster centroid. Until the sum can't be reduced further, it moves sensor node between clusters. A set of clusters that are well-separated and compact as possible is resulted.

$$
I\left\{c_{1}, c_{2}, \ldots . c_{n}\right\}=\sum_{k=1}^{n} \sum_{x_{i} \in c_{k}} D\left(x_{i}-c_{k}\right)
$$

Where, $\mathrm{D}\left(x_{i}-c_{k}\right)$ is the Euclidean distance between $x_{i}$ and $c_{k}$. With this above equation (5), the sensor node which is the least distance from centroid will be allocated for each clusters as Backup cluster head.

The centroid of the nodes is calculated using the below equation (6)

$$
\operatorname{Cent}_{i}=\frac{\operatorname{Dist}_{i, \operatorname{sink}}+\sqrt{\left(X_{i}-X_{j}\right)^{2}+\left(Y_{i}-Y_{j}\right)^{2}}}{N}
$$

Here Dist $_{i, \operatorname{sink}}$ denotes the node i's distance between the node and BS, $\left(X_{i}, Y_{i}\right)$ and $\left(X_{j}, Y_{j}\right)$ are the $\mathrm{X}, \mathrm{Y}$ positions of the nodes $\mathrm{I}, \mathrm{j}, \mathrm{N}$ is the value of total number of nodes in the network. 
In the first phase, the respective positions are detected by the nodes with the help of GPS, and nodes within the respective communication range are informed with the transmission of a Hello packet, including the individual ID and location (Xi, Yi). The respective neighbor's data is stored within a table named as neighbors table by the nodes after the arrival of the 'Hello' packets from neighbors. Later, the data is counted and stores the number of the received packets within variable $\mathrm{n}$ for identifying the several neighbors. The respective individual positions are informed to the nodes with the help of GPS and known BS locations. Accordingly, the calculation of the distance among the BS as well as the nodes is done. The nodes estimate the current delay between the nodes by probing the links. The individual estimation values are sent by the nodes towards the respective neighbors via an advertisement packet to calculate such multiple factors. The nodes know the individual estimation values regarding individual neighbors. Therefore, the individual estimation values are compared by them with other values, and the selection of the $\mathrm{CH}$ is made when a node fulfills the abovementioned conditions. For $\mathrm{BKCH}$ selection, the nodes start estimating the centroid and the probability values in the next round. The nodes share these estimations with their non- $\mathrm{CH}$ neighbors and compare the estimations. The nodes which satisfy the criteria are selected as $\mathrm{BKCH}$. The result of the $\mathrm{CH}$ selection \& the $\mathrm{BKCH}$ selection will be updated to the sensor nodes using the beacon messages. The following figure 3 represents the beacon format for eligible $\mathrm{Ch}$ and $\mathrm{BKCH}$ selection.

\begin{tabular}{|l|l|l|}
\hline PKT TYPE & CH-ID & BKCH-ID \\
\hline
\end{tabular}

\section{Fig. 3. Beacon format}

\subsection{Routing phase:}

Intra-cluster routing: After selecting the $\mathrm{CHs}$ and $\mathrm{BKCHs}$, the next stage is routing. The sensor nodes must deliver the sensed data to BS in anyways. Usually, in clustered environments, the information out of the member nodes is aggregated by the $\mathrm{CHs}$. This information is then forwarded to the BS. The traffic within the network, as well as the congestion, is reduced by this approach drastically. By taking advantage of this aggregation technique, in our protocol, the elected $\mathrm{CHs}$ aggregate the information from the member nodes using the path discovered by the routing protocol.

Inter-cluster routing: In inter-cluster routing, the $\mathrm{CH}$ nodes share the aggregated data to the BS node for further analysis. In 
traditional clustered environments, the $\mathrm{CH}$ node directly establishes the connection to the BS irrespective of the situation. Suppose multiple $\mathrm{CH}$ nodes are connected with the BS concurrently, the overhead of the BS is increased. To avoid that, we introduce AGGREGATOR nodes (AG), which aggregate the $\mathrm{CH}$ nodes' data and share it with BS. By this, the overhead in BS is avoided. To establish the connection and select the best relay nodes between AG nodes and $\mathrm{CH}$ nodes, the routing protocol's relay selection procedure is modified by PSO functions. This PSO method calculates the nodes' fitness and selects the best fit relay node based on Particle Best (PBEST) and Global best (GBEST) values. The particle best $\&$ the global best values are updated as follows

$$
\text { Pbest }_{i}=\left\{\begin{array}{lr}
P_{i} & \text { if } \left.\left(\text { fitness }\left(P_{i}\right)<\text { fitness }_{\text {Pbest }}\right)\right) \\
\text { Pbest }_{i} & \text { otherwise }
\end{array}\right.
$$

The global best value can be updated as follows

$$
\text { Gbest }_{i}=\left\{\begin{array}{lr}
P_{i} & \text { if }\left(\text { fitness }\left(P_{i}\right)<\text { fitness }(\text { Gbest })\right) \\
\text { Gbest } & \text { otherwise }
\end{array}\right.
$$

ModPSO relay selection: The PSO routing technique's development is done based on the centralized technique, and the main objective is presented below. The bootstrapping procedure is performed by every sensor node and AG initially, in which the exclusive IDs are allocated to each node by BS. Later, the individual IDs are broadcasted by the sensor nodes as well as the AGs. Every AG collects the overall sensor nodes' IDs and the CHs with the high and low communication range. The setup phase, in addition to the steadystate phase, is included in the network setup. $A G$ is used to set up the final route within the setup phase with this technique's help. The information regarding the respective succeeding hop $\mathrm{CH}$ is provided to the overall CHs during the route setup phase to AG. The data out of the respective member sensor nodes is received, aggregated, and forwarded towards AG by $\mathrm{CHs}$ within every round.

Since our aim is fault tolerant energy efficient routing, the PSO's fitness function is modified to choose the nodes based upon the fitness function in addition to residual energy. The modified fitness function of the proposed protocol is shown in equation (7),

$$
\begin{aligned}
& \text { fitness }(i)= \sqrt{\left(X_{i}-X_{j}\right)^{2}+\left(Y_{i}-Y_{j}\right)^{2}}+ \\
& \frac{E_{r e s}}{E_{\text {con }}}
\end{aligned}
$$

Here, in $\frac{E_{\text {res }}}{E_{\text {con }}}, E_{\text {res }}$ is the residual energy of the nodes, $E_{c o n}$ denotes the consumed energy level of the nodes. This estimation gives the lifetime of the node as result and it is considered for fitness calculation. $E_{c o n}$ Can be calculated based on the number of packets 
received, the energy consumed during receiving. It can be expressed as follows in equation (8)

$$
E_{\text {con }}=N P_{L} * E_{r}+E_{T}
$$

Here, $N P_{L}$ represents the total number of data packets received at the data length of $\mathrm{L}, E_{r}$ is the energy consumption of the sensor nodes due to receiving and $E_{T}$ denotes the energy consumed due to transmitting the data to the next hop nodes.

Due to this modification, the relay node with high lifetime is selected. This improves the data delivery rate in long duration communications.

Since our objective is to find out the optimal relay nodes for efficient data transmission. This can be possible by evaluating the fitness of the sensor nodes based on the distance and the energy availability at each sensor node. Therefore, it can be expressed as follows in equation (9)

$$
\begin{array}{r}
\text { Objective: Optimal relay }= \\
\operatorname{best}\{\operatorname{fitness}(n) \mid n \in N\}
\end{array}
$$

Fault tolerance: If the $\mathrm{CH}$ of a cluster is failed during the steady phase then the member nodes select $\mathrm{BKCH}$ toward the $\mathrm{BS}$ to continue its operation. The failure can be detected by a sensor node if it does not reach the $\mathrm{CH}$ during data transmission. After that the member node joins a $\mathrm{BKCH}$ the data transmission resumes until the end of the transmission.

\subsection{Algorithm setup for FTOR-ModPSO:}

\section{Algorithm for $\mathrm{CH}$ selection}

\section{Input:}

$d_{i, \operatorname{sink}}=$ distance between the nodes and BS; $E_{\text {res }}=$ residual energy of node $\mathrm{i}$

$d e_{i}=$ delay of the node $\mathrm{i}$;

$\operatorname{Pro}_{i}=$ probability of node $\mathrm{i} ;$

Output: cluster head list $\mathrm{CH}[\mathrm{i}]$

\#\#

Step 1: For all the nodes $n$

Step 2: Calculate $d_{i, \sin k}$

Step 3: Calculate $d e_{i}$

Step 4: Estimate Pro $_{i}$

Step 5: If $\left(d_{i, \sin k}(\mathrm{n})<d_{i, \operatorname{sink}} \mathrm{n}+1\right) \& \& \operatorname{Pro}_{i}(\mathrm{n})>\operatorname{Pro}_{i}$ $(\mathrm{n}+1))$

Step 6: If $\left(d e_{n}<d e_{n+1}\right)$

Step 7: $\mathrm{CH}[\mathrm{i}]=\mathrm{n}$

Step 8: Else

Step 9: $\mathrm{CH}[\mathrm{i}]=\mathrm{n}+1$

Step 10: End for

Step 11: For all the nodes $n$

Step 12: If $\mathrm{n} \varepsilon \mathrm{CH}[\mathrm{i}]$ 
Step 14: Else

Step 15: Joins with the nearest $\mathrm{CH}$ as cluster member

\section{Algorithm for BKCH selection}

\section{Input:}

$d_{i, \text { sink }}=$ distance between the nodes and BS;

$d_{i, j}=$ distance between the nodes I and $\mathrm{j}$;

Cent $_{i}=$ centroid of node $\mathrm{I} ;$

$\operatorname{Pro}_{i}=$ probability of node $\mathrm{i} ;$

Output:

$\mathrm{BKCH}[\mathrm{i}]=$ Backup cluster head list

\#\#

Step 1: For all the nodes $n$

Step 2: Calculate $d_{i, \sin k}$

Step 3: Estimate $\mathrm{Pro}_{i}$

Step 4: If $\left(d_{i, \operatorname{sink}}(\mathrm{n})+d_{i, j}(\mathrm{n})\right)<\left(d_{i, \operatorname{sink}}(\mathrm{n})+d_{i, j}\right.$ $(\mathrm{n}+1))$

Step 5: If $\left(\operatorname{Pro}_{i}(\mathrm{n})>\operatorname{Pro}_{i}(\mathrm{n}+1)\right)$

Step 6: $\mathrm{CH}[\mathrm{i}]=\mathrm{n}$

Step 7: Else

Step 8: $\mathrm{CH}[\mathrm{i}]=\mathrm{n}+1$

Step 9: End for 


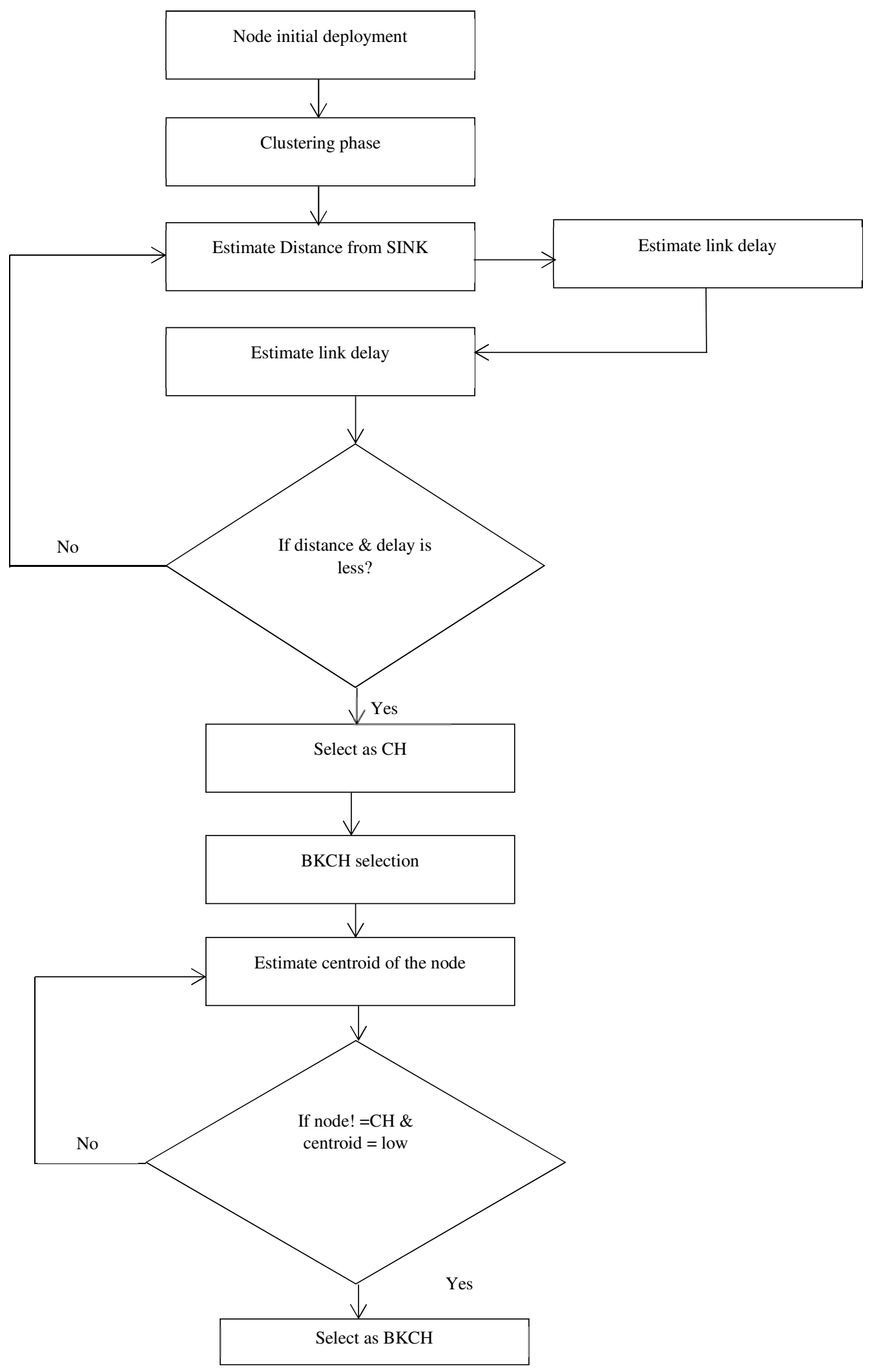

Fig. 4. Flowchart of $\mathrm{CH}$ and $\mathrm{BKCH}$ selection 


\section{Algorithm for ModPSO relay selection}

\section{Input:}

$P_{i}=$ total participating particles

$f_{i}=$ fitness of the particles

$E_{\text {res }}=$ residual energy; $E_{\text {con }}$ consumed energy; $L T_{i}=$ lifetime of node I;

$P_{\text {best }}=$ particle best of node I; $G_{\text {best }}=$ Global best of node I;

Output:

$\mathrm{NR}=$ next relay

\#\#

Step 1: For all the nodes $n$

Step 2: Calculate $f$ i of all particles $P_{i}$

Step 3: Estimate $E_{\text {res }}, E_{\text {con }}$

Step 4: Calculate dist $_{i}$

Step 5: Calculate $L T_{i}=\frac{E_{\text {res }}}{E_{\text {con }}}$

Step 6: $\quad P_{\text {best }}=L T_{i}$

Step 7: $\quad G_{\text {best }}=\left\{\max \left[P_{\text {best }}\right]\right\}$

Step 8: End

Step 9: For all the nodes $n$

Step 10: If $\left(G_{\text {best }} P_{i}>G_{\text {best }} P_{i+1}\right)$

Step 11: NR $=P_{i}$

Step 12: Else

Step 13: $\quad \mathrm{NR}=P_{i+1}$
Step 14: End for

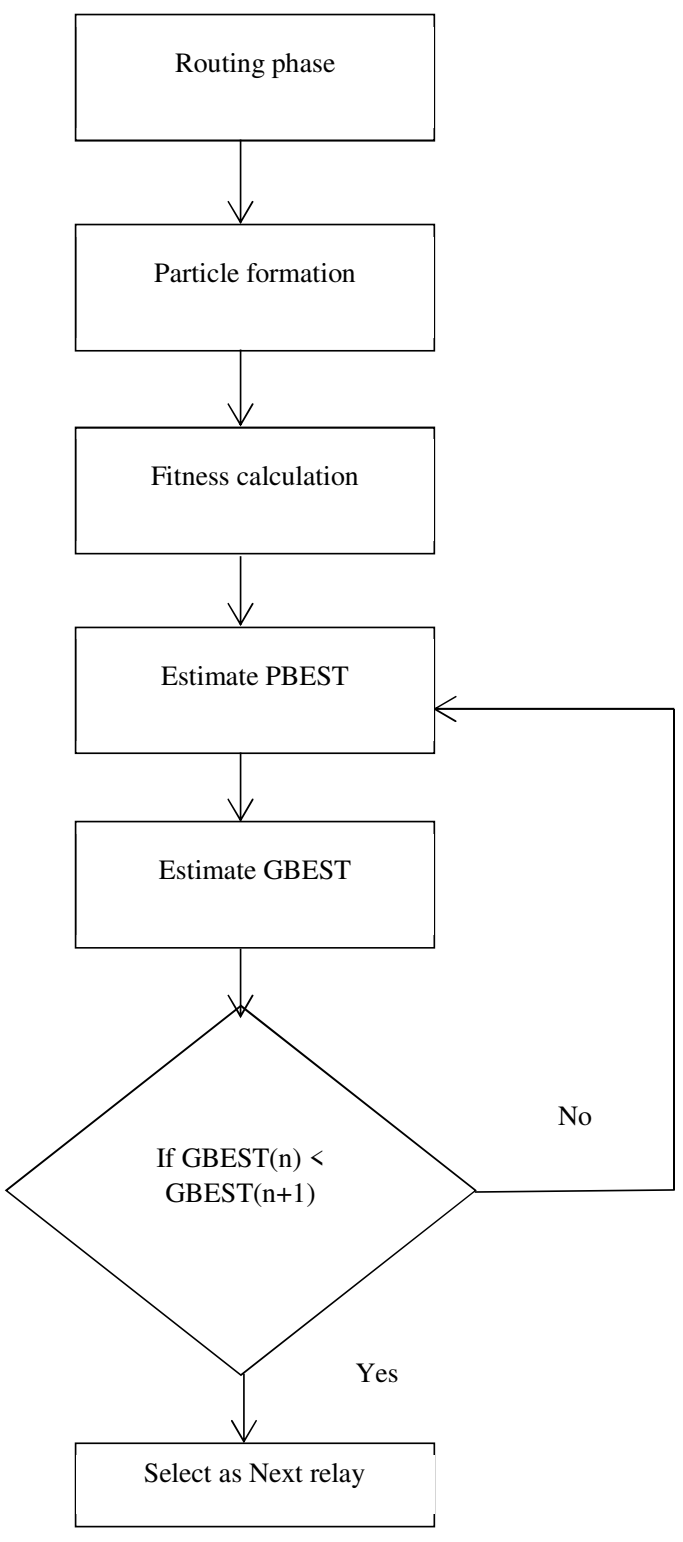

Fig. 5. Flowchart of Modified PSO relay selection 


\section{Results and Discussion}

For both routing failure and $\mathrm{CH}$ failure, fault tolerance is provided by the proposed mechanism that solely depends on a modified PSO algorithm to choose BKPCHs and an optimal $\mathrm{CHs}$ for fault tolerance. At the $\mathrm{CH}$ level, the aggregation of data is performed and aggregators utilize for reducing the congestion and communication overhead. In this section, the proposed technique of FTOR-ModPSO is evaluated and the efficiency is validated by comparing it with the two existing techniques such as EMLEACH [26], QEBSR [27], QOS-IHC [28], and ML-SEEP [29]. To implement these methods, a similar simulation environment is utilized. For the simulation of energy parameters, the NETWORK SIMULATOR2 is used. Different parameters like packet delivery ratio, overhead, throughput, energy consumption, and an end-to-end delay are analyzed based on the simulation results.

\subsection{Simulation Environment}

A square area of $1000 \times 500 \mathrm{~m}$ is considered to perform the simulation in which the deployment of the sensors is done in a random way. The location of a sink node is within the center of a sensing area where the deployment of the nodes is performed accidentally. Table 1 demonstrates the network parameters. Table 2 shows the performance comparison between different protocols in a $1000 \mathrm{mx} 500 \mathrm{~m}$ sensor field.

Table1: Simulation parameters

\begin{tabular}{|l|l|}
\hline PARAMETER & VALUE \\
\hline Application traffic & CBR \\
\hline Transmission rate & 1024 bytes/ 0.5ms \\
\hline Radio range & $250 \mathrm{~m}$ \\
\hline Packet length & 1024 bytes \\
\hline Routing Protocol & AODV \\
\hline Simulation time & $100 \mathrm{~s}$ \\
\hline Number of sensor nodes & 50 \\
\hline Area length & $1000 \mathrm{x} 500$ \\
\hline Transmission Protocol & $\mathrm{UDP}$ \\
\hline Initial Energy & $100 \mathrm{j}$ \\
\hline BKPCH & 4 \\
\hline Clusters & 4 \\
\hline
\end{tabular}

\subsection{Graphical analysis}

Table2: A performance comparison between different protocols in a $1000 \mathrm{~m}$ x $500 \mathrm{~m}$ sensor field

\begin{tabular}{|l|l|l|}
\hline Protocol & Lifetime & $\begin{array}{l}\text { Total Energy } \\
\text { Consumption }\end{array}$ \\
\hline
\end{tabular}




\begin{tabular}{|l|l|l|}
\hline $\begin{array}{l}\text { EM-LEACH } \\
{[26]}\end{array}$ & 88.578 & 11.422 \\
\hline QEBSR [27] & 90.334 & 9.666 \\
\hline $\begin{array}{l}\text { QOS-IHC } \\
{[28]}\end{array}$ & 90.902 & 9.098 \\
\hline MLSEEP [29] & 91.866 & 8.134 \\
\hline $\begin{array}{l}\text { FTOR- } \\
\text { ModPSO }\end{array}$ & 92.714 & 7.286 \\
\hline
\end{tabular}

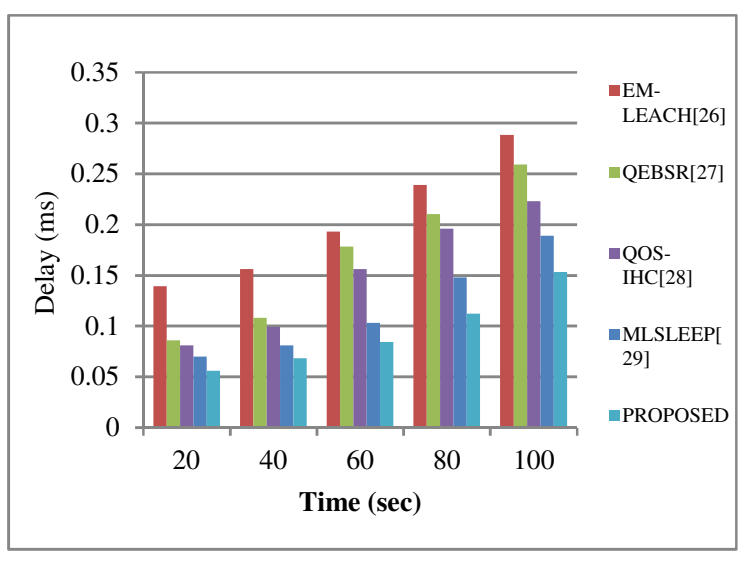

Fig. 6. Analysis of Delay

The data should be aggregated and delivered to the targeted receivers on time for further escalation. In the existing protocols, the absence of the fault tolerance and improper forwarder node selection leads to retransmissions often, which results in a high delay between the sensor nodes. The proposed protocol avoids unnecessary factors such as high hops and high data traffic that affect the data delivery between the nodes. The result shows that the proposed protocol has a lesser delay than previously proposed protocols in the given time. Figure 6 shows the end-to-end delay of the overall network.

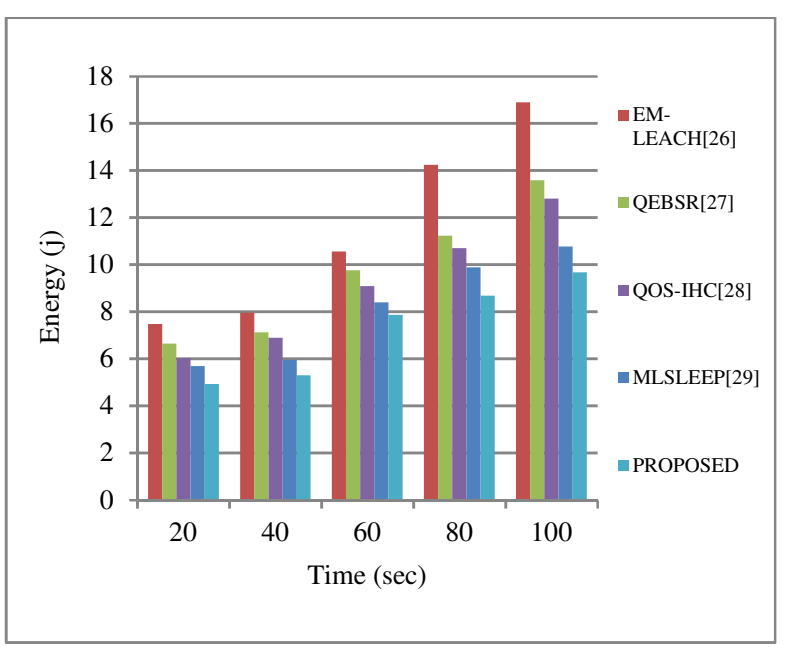

Fig. 7. Energy Consumption

Energy is vital for the sensor nodes for continuous operation. Sensor networks are limited energy networks. The optimized usage of energy gives a longer lifetime. There are no proper data aggregation techniques presented in the existing protocols that reduce the communication overhead, which increases the overall energy consumption ratio. Our proposed method's fault tolerance mechanism alleviates factors such as retransmission and wrong path and improves overall energy consumption using effective data aggregation techniques. The result proves that the energy consumption rate is comparatively lesser than the conventional protocols. Figure 7 shows the graphical representation of energy consumption. 


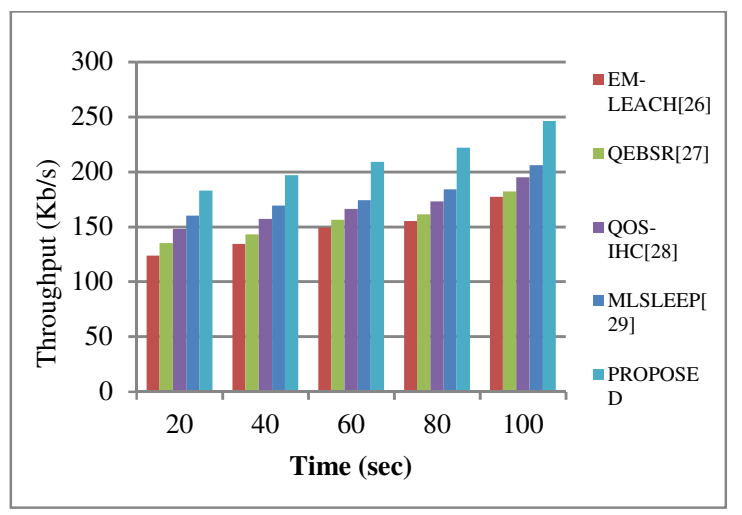

Fig. 8. Network Performance

The division of clusters and the fine selection of $\mathrm{CHs}$ using multiple relevant parameters make CHs easily accessible by the member nodes. In the existing works, the unexpected congestion due to the sensor nodes' burst traffic is not addressed effectively. Thus, the throughput ratio is affected drastically. Aggregator nodes and PSO usage ensure seamless data delivery across the network, helping the proper data delivery without much interruption. The result proves that the proposed approach delivers the data more reliable than the previously proposed protocols. Figure 8 represents the network performance.

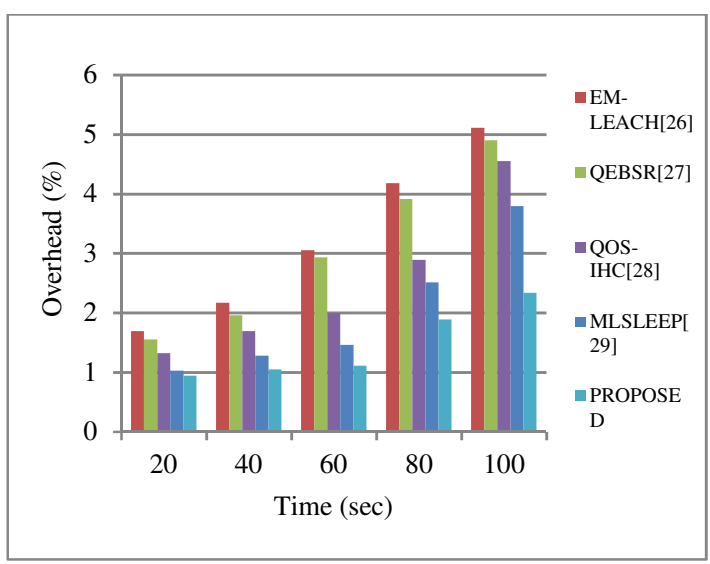

Fig. 9. Routing overhead

The amount of control packets required for the network operation decides the network's overhead, representing in figure 9. In the previously proposed works, the number of retransmissions due to link breakage and improper relay node selection is quite high. Also, the available methods to handle route failure require additional control packets, increasing the network's overhead. Frequent retransmission due to the network failure requires additional usage of control packets, which affects the overhead. In our proposed protocol, the fault tolerance mechanism ensures break-free communication. Also, it provides a backup mechanism for uninterrupted transmission, which reduces the need for additional control packets and reduces the overhead. 


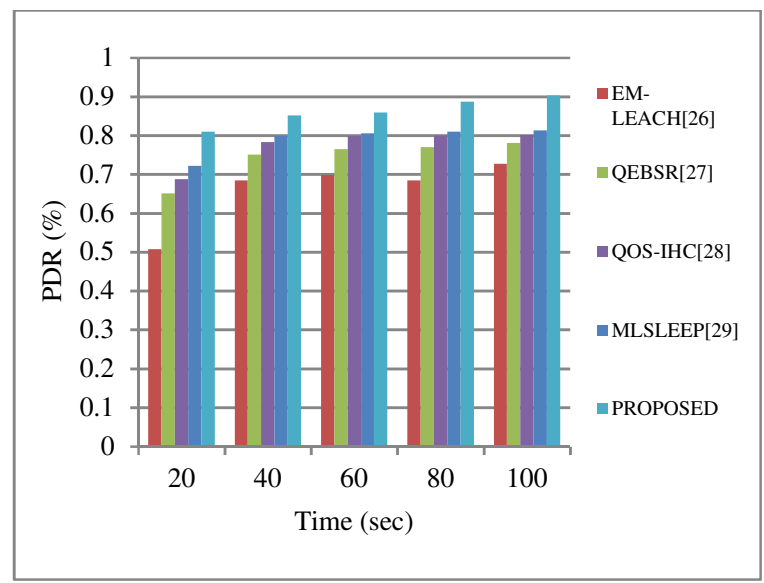

Fig. 10. Packet Delivery Ratio

Selecting an uninterrupted and shortest routing decision improves the data delivery ratio, and it shows in figure 10 . In the existing works, the data delivery rate is seriously affected by $\mathrm{CH}$ failure and link failure. There are no proper fault tolerance mechanisms available that handle the failure and avoid the retransmissions. In our proposed approach, the data packets are transmitted through the globally best paths identified by the PSO algorithm. The result proves that the proposed approach provides more PDR results than traditional protocols.

\section{Conclusion}

It is quite complicated to provide fault tolerance as the energy resources of sensor nodes is restricted. Clustering and routing are the 2 phases included in the fault tolerance provided by this paper. The selection of $\mathrm{CHs}$ and BKPCHs is made depending upon a few suitable metrics within the initial phase. From cluster member nodes to the $\mathrm{CH}$, the data is transmitted in the second phase. The $\mathrm{CH}$ nodes share the data to AGGREGATORs before sending it to $\mathrm{BS}$ within the intercluster routing. A significant role is played by clustering and routing play by allotting the fault tolerance towards the network. It is shown in the simulation outcomes that this approach concerning end-to-end delay outperforms the previous approaches, throughput, average energy consumption, routing overhead, as well as packet delivery rate percentage.

\section{Declarations:}

Funding: Not applicable

\section{Conflicts of interest:}

\section{Author name: POGULA SREEDEVI}

Title: FTOR-ModPSO: A fault tolerance and an optimal relay node selection algorithm for wireless sensor networks using Modified PSO

No financial support from private or government institutions

Under supervisor

Dr S Venkateswarlu

Professor in Koneru Lakshmaiah Educational Institution

\section{Availability of data and material:}

Not applicable 


\section{Code availability:}

Available

\section{Authors' contributions:}

Not applicable

\section{References:}

[1]. C.-Y. Chong, S.P. Kumar, (2003). Sensor networks: evolution, opportunities, and challenges, Proc. IEEE 91, 1,247-1,256.

[2]. I. F. Akyildiz, W. Su, Y. Sankarasubramaniam, and E. Cayirci, (2002). "Wireless sensor networks: A survey," Comput. Netw., vol. 38, no. 4, pp. 393-422.

[3]. K. Lin, C. F. Lai, X. Liu, and X. Guan, (2012). "Energy efficient routing with node compromised resistance in wireless sensor networks," Mobile Netw. Appl., vol. 17, pp. 75-89.

[4]. K. A. Darabkh, N. J. Al-Maaitah, I. F. Jafar, and K. Ala'F, (2017). "EACRP: A novel energy-aware clustering and routing protocol in wireless sensor networks," Comput. Elect. Eng., vol. 72, pp. 702-718.

[5]. J. Chang and L. Tassiulas, (2004). "Maximum lifetime routing in wireless sensor networks," IEEE/ACM Trans. Netw., vol. 12, no. 4, pp. 609-619, Aug.
[6]. A. A. Ahmed and Y. Mohammed, (2007) “A survey on clustering algorithms for wireless sensor networks, Elsevier," Comput. Commun., vol. 30, pp. 2826-2841.

[7]. T. He, J. A. Stankovic, C. Lu, and T. Abdelzaher, (2003). "SPEED: A stateless protocol for real-time communication in sensor networks," in Proc. $23^{\text {rd }}$ Int. Conf. Distrib. Comput. Syst., pp. 46-55.

[8]. E. Felemban, C. G. Lee, and E. Ekici, (2006). "MMSPEED: Multipath multispeed protocol for QoS guarantee of reliability and timelines in wireless sensor networks," IEEE Trans. Mobile Comput., vol. 5, no. 6, pp. 738-754, Jun.

[9]. X. Huang and Y. Fang, (2008). "Multiconstrained QoS multipath routing in wireless sensor networks," J. Wireless Netw., vol. 14, no. 4, pp. $465-478$

[10]. X. Song, C. Wang, and J. Pei, (2012). "ASenNet: A multiple QoS metrics hierarchical routing protocol based on swarm intelligence optimization for WSN," in Proc. IEEE Int. Conf. Inf. Sci. Technol., Hubei, China, Mar. 2325, pp. 531-534.

[11]. W. Cai, X. Jin, Y. Zhang, K. Chen, and R.Wang, (2006). "ACO based QoS routing algorithm for wireless sensor networks," in Proc. 3rd Int. 
Conf.Ubiquitous Intell. Comput., LNCS, pp. 419-428.

[12]. M. A. Adnan, M. A. Razzaque, I. Ahmed, and I. F. Isnin, (2014). "Biomimic optimization strategies in wireless sensor networks: A survey," Sensors, vol. 14, pp. 299-345.

[13]. S. Hadim and N. Mohamed, (2006). "Middleware: Middleware challenges and approaches for wireless sensor networks," IEEE Distrib. Syst. Online, vol. 7, no. 3, Mar., pp. 1-23.

[14]. S. Yi, P. Naldurg, and R. Kravets, (2001). "Security-aware protocol for wireless ad-hoc networks," in Proc. 2nd ACM Int. Symp. Mobile Ad Hoc Netw. Comput., Long Beach, CA, USA, pp. 299-302.

[15]. F. Khan, (2014). "Secure communication and routing architecture in wireless sensor networks," in Proc. IEEE 3rd Global Conf. Consum. Electron., pp. 647650.

[16]. B. H. Calhoun, D. C. Daly, N. Verma et al., (2005). "Design considerations for ultra-low energy wireless microsensor nodes," IEEE Transactions on Computers, vol. 54, no. 6, pp. 727740.

[17]. H. Harb and A. Makhoul, (2018). "Energy-efficient sensor data collection approach for industrial process monitoring," IEEE
Transactions on Industrial Informatics, vol. 14 , no. 2, pp. 661672.

[18]. D. Kumar, T. C. Aseri, and R. B. Patel, (2009). "EEHC: energy efficient heterogeneous clustered scheme for wireless sensor networks," Computer Communications, vol. 32, no. 4 , pp. 662-667.

[19]. D. Sharma and A. P. Bhondekar, (2018). "Traffic and energy aware routing for heterogeneous wireless sensor networks," IEEE Communications Letters, vol. 22, no. 8, pp. 1608-1611.

[20]. S. Dutt, S. Agrawal, and R. Vig, (2018). "Cluster-head restricted energy efficient protocol (CREEP) for routing in heterogeneous wireless sensor networks," Wireless Personal Communications, vol. 100, no. 4, pp. 1477-1497.

[21]. S. Tanwar, S. Tyagi, N. Kumar, and M. S. Obaidat, (2019). "LA-MHR: learning automata based multilevel heterogeneous routing for opportunistic shared spectrum access to enhance lifetime of WSN," IEEE Systems Journal, vol. 13, no. 1, pp. 313-323.

[22]. Z. Hong, L. Yu, and G.-J. Zhang, (2013). "Efficient and Dynamic Clustering Scheme for Heterogeneous Multi-level Wireless Sensor 
Networks," Acta Automatica Sinica, vol. 39 , no. 4 , pp. $454-460$.

[23]. Z. Hong, R. Wang, and X. Li, (2016). "A clustering-tree topology control based on the energy forecast for heterogeneous wireless sensor networks," IEEE/CAA Journal of Automatica Sinica, vol. 3, no. 1, pp. 68-77.

[24]. M. Faheem and V. C. Gungor, (2018). "Energy efficient and QoS aware routing protocol for wireless sensor network- based smart grid applications in the context of industry 4.0," Applied Soft Computing, vol. 68, no. 7, pp. 910-922.

[25]. A. K. Mishra, R. U. Rahman, R. Bharadwaj, and R. Sharma, (2015). "An enhancement of PEGASIS protocol with improved network lifetime for wireless sensor networks," in 2015 IEEE Power, Communication and Information Technology Conference (PCITC), pp. 142-147, Bhubaneswar, India.

[26]. Sara AI-Sodairi, Ridha Ouni, (2018). "Reliable and Energy-Efficient MultiHop LEACH-Based Clustering Protocol for Wireless Sensor Networks", Sustainable Computing:
Informatics and Systems, Volume 20, Pages 1-13. https://doi.org/10.1016/j.suscom.201 8.08.007.

[27]. M. Rathee, S. Kumar, A. H. Gandomi, K. Dilip, B. Balusamy and R. Patan, (2019). "Ant Colony Optimization Based Quality of Service Aware Energy Balancing Secure Routing Algorithm for Wireless Sensor Networks," in IEEE Transactions on Engineering Management, doi: 10.1109/TEM.2019.2953889.

[28]. Parvinder Singh, Rajeshwar Singh, (2019). "Energy-Efficient QoSAware Intelligent Hybrid Clustered Routing Protocol for Wireless Sensor Networks", Journal of Sensors, vol. 2019, Article ID 8691878, 12 pages. https://doi.org /10.1155/2019/8691878.

[29]. Robinson, Y.H., Julie, E., Kumar, R. (2019). Probability-based cluster head selection and fuzzy multipath routing for prolonging lifetime of wireless sensor networks. Peer-toPeer Netw. Appl. 12, 1061-1075. https://doi.org/10.1007/s12083-01900758-8. 